\title{
Propuesta de plan de conservación preventiva para proyectos de muralismo urbano. El caso de Vigo, ciudad de color
}

\author{
Andrea Fernández Arcos
}

Resumen: Son numerosas las ciudades que se están dejando subyugar por el cambio en su fisionomía que proporcionan los grandes murales urbanos, y también algunas empiezan a conocer los problemas que plantea su conservación. En 2015 el Ayuntamiento de Vigo se sumó a esta tendencia con el objetivo de transformar el feísmo urbanístico en un museo al aire libre.

Este estudio pretende analizar los problemas de conservación a los que se enfrentan estas obras por su ubicación y por las particulares condiciones ambientales de la ciudad, pero también desde la óptica de los nuevos materiales con los que han sido creadas y la perspectiva de conservación que sus autores tienen sobre ellas, con el fin de crear un plan de conservación preventiva que facilite su preservación.

Palabras clave: pintura mural, arte urbano, conservación, conservación preventiva, patrimonio cultural

\section{Proposal for a preventive conservation plan for urban mural projects. The case of vigo, city of color}

Abstract: Many cities are being subjugated by the change in their physiognomy provided by large urban murals and some are beginning to understand the problems posed by their conservation. In 2015, Vigo City Council joined this trend with the aim of transforming urban feism into an open-air museum.

This study aims to analyze the conservation problems faced by these works due to their location and the particular environmental conditions of the city, but also from the perspective of the new materials with which they have been created and the conservation perspective that their authors have on them, in order to create a preventive conservation plan that facilitates their preservation.

Keyword: mural painting, street art, conservation, preventive conservation, cultural heritage

\section{Proposta de plano de conservação preventiva para projetos de muralismo urbano: 0 caso da cidade de Vigo}

Resumo: Muitas cidades estão a ser subjugadas por uma mudança fisionómica proporcionada pelos grandes murais urbanos, e algumas também começam a conhecer os problemas colocados na sua conservação. Em 2015, a Câmara Municipal de Vigo aderiu a esta tendência com o objetivo de transformar o urbanismo num museu ao ar livre.

Este estudo tem como objetivo analisar os problemas de conservação que estas obras enfrentam devido à sua localização e às condições ambientais particulares da cidade, mas também a perspetiva dos novos materiais com os quais foram criadas e a perspetiva da conservação que os seus autores têm sobre elas, a fim de se criar um plano de conservação preventiva que facilite a sua preservação.

Palavras-chave: pintura mural, arte urbana, conservação, conservação preventiva, património cultural 


\section{Introducción}

En 2015 el Ayuntamiento de Vigo promovió la creación de un museo al aire libre compuesto por pinturas murales, con gran acogida por parte de los ciudadanos. Este texto pretende diseñar una estrategia de trabajo que facilite su preservación.

Para los conservadores restauradores no resulta novedoso realizar un plan de conservación preventiva para un grupo heterogéneo de obras como éste. Lo que sí se considera una particularidad de este estudio es que pretende crear un plan para un conjunto de obras de distintos artistas, con sus particularidades, repartidas al aire libre en un área de $109 \mathrm{~km}^{2}$ aproximadamente.

Si el planteamiento es exitoso, esta propuesta permitirá mejorar la conservación de los murales pertenecientes al proyecto Vigo. Ciudad de color, optimizando la inversión de recursos públicos. Además, las pautas de trabajo podrán ser extrapolables a otros conjuntos murales, cada vez más presentes en las ciudades.

\section{Objetivos}

El principal objetivo de este trabajo es diseñar un plan de conservación preventiva para el conjunto de murales pertenecientes al proyecto Vigo. Ciudad de color. También pretende conocer el contexto en que han sido creados los murales, determinar los factores de alteración decisivos para su conservación, conocer las expectativas de conservación que tienen los distintos actores vinculados al proyecto y reflexionar sobre el papel de los conservadores restauradores en la conservación de este tipo de conjuntos.

\section{Vigo. Ciudad de color}

El proyecto surgió con la aparición en 2014 de un nuevo solar en la Calle del Príncipe. Los ciudadanos expresaron un especial malestar al quedar expuesta una llamativa medianera en una de las vías que más turistas recibe todos los días. Para paliar el daño visual desde el Ayuntamiento se tomó la decisión de taparla con una enorme lona pintada con la obra "Fiestra" del surrealista gallego Lugrís.

La acogida entre los ciudadanos fue extraordinaria, aunque finalmente se construyó un edificio en el solar y la lona fue retirada; pero la gran aceptación convirtió este primer mural en el detonante de un nuevo proyecto. En agosto de 2014 la prensa recogía que "La iniciativa costó 20.000 euros y se repetirá en más de cien medianeras repartidas por toda la ciudad, incluidas las parroquias (...) con el fin de embellecer la ciudad" (Atlántico Diario 2014).

Este mural supuso el primer paso de Vigo. Ciudad de color, que tendría como objetivo mejorar el paisaje urbano con graffiti y otras especialidades pictóricas, según se indica en su propia web [1]. Abel Caballero, alcalde de la ciudad, aseguró que así se conseguirían recuperar "(...) zonas afeadas por un urbanismo nefasto, haciendo concursos y otras iniciativas con pintores muralistas" (Pastoriza 2014).

Esta desafortunada planificación de la urbe a la que se refería el alcalde tiene como uno de sus máximos exponentes las medianeras de los edificios. Lo habitual es que estas medianeras se encuentren sin remates estéticos dejando el material de construcción a la vista, o bien tapadas tan sólo con cemento, con pintura plana, con chapa metálica, espuma de poliuretano o tela asfáltica.

Hasta ahora algunas de ellas habían sido utilizadas como soporte publicitario e incluso como soporte de intervenciones artísticas. $Y$ ya hay un buen número de ciudades que, como Vigo, se han sumado a esta misma iniciativa.

Estamos pues, ante una fórmula no original en la propuesta, pero si en la cantidad de obras en una misma ciudad, puesto que el propio alcalde ha anunciado la voluntad de convertir Vigo en la ciudad europea con mayor número de murales (Méndez 2017).

En la web diseñada por el ayuntamiento para dar difusión a este proyecto se explican sus objetivos: además de promover el street art, esta iniciativa pretende la creación de un museo al aire libre mediante la transformación del feísmo urbano en arte "(...) gestionando actividades y actuaciones culturales complementarias, convirtiendo la participación ciudadana en un pilar del cambio artístico de la ciudad".

El principal objetivo de Vigo. Ciudad de color era paliar la estética desagradable causada por algunos inmuebles, pero con el tiempo se han sumado otros: se trata de un proyecto integrador, puesto que han sido incluidos barrios de distinta condición, del centro o periféricos. Tanto es así que los vecinos del barrio de Ribadavia solicitaron un mural al Ayuntamiento tras una etapa de mejora de infraestructuras. El Ayuntamiento accedió, creando un mural sobre una medianera descubierta tras la demolición de una infravivienda (Ponce 2019).

Vigo. Ciudad de color pretende también atraer a artistas reconocidos así como respaldar a artistas de la ciudad, ya sean pintores consolidados (incluso en otros ámbitos, como pintura de caballete) o nuevos valores. $Y$ hay que destacar que se ha convertido en un nuevo atractivo turístico para la ciudad.

De este modo, en 2015 se realizaron 14 murales, otros 14 en 2016, 15 en 2017 y 15 en la edición de 2018. Hasta 2018 se pintaron 58 murales repartidos por toda la ciudad y su creación ha supuesto ya un total de $1.049 .626,23 €$ procedentes del erario público [2]. Para el año 2019 se anunció en la prensa la creación de 14 nuevos murales por un importe de $280.176 €$ (Prego 2018). 
El Ayuntamiento ha desarrollado otras actividades alrededor de esta iniciativa, como talleres artísticos o actuaciones musicales (Ponce 2018).

En resumen, es un proyecto muy ambicioso que pretende un gran impacto sobre los ciudadanos y que implica un gran volumen presupuestario.

\section{Antecedentes}

Las pintadas en las paredes, que en un principio se percibían como un acto de vandalismo, han adquirido nuevos valores para la sociedad, evolucionando en distintas las expresiones artísticas sobre todo tipo de soportes urbanos.

Algunos autores han reflexionado ya sobre la necesidad de conservación del arte urbano, como Mata Delgado (2013), Ciancabilla (2015), o Sánchez Pons et al. (2015). Chatzidakis (2018:99) sitúa el origen de este interés en Reino Unido, señalando a la sociedad como su detonante y en nuestro país hay que destacar necesariamente el intenso trabajo que el Grupo de Trabajo de Arte Urbano del Ge-IIC, creado en 2015, viene desarrollando en los últimos años.

Inicialmente el arte urbano no nacía con pretensión de permanencia: la irregularidad y clandestinidad de su factura lo ligaba de forma indisoluble a un carácter efímero y por el riesgo de ser descubiertos durante su realización, de formato normalmente pequeño. Nada que ver con las grandes medianeras que necesitan de cierta infraestructura para ser pintadas, como es este el caso. Sin embargo en términos de conservación presentan numerosos puntos en común, como los materiales empleados o los riesgos a los que se ven expuestos.

Luque Rodrigo investiga cómo segestionala perdurabilidad de aquellas obras encargadas por las instituciones públicas (2015:13). En el caso de Vigo veremos que el punto de partida es la voluntad del propio ayuntamiento.

\section{Descripción material}

En Vigo. Ciudad de color tanto artistas como espacios fueron elegidos por una Comisión técnica creada para cada una de las fases del proyecto. En esta comisión, nombrada por la Concejalía de Patrimonio Histórico, estarían representadas las asociaciones culturales de la ciudad y técnicos municipales especializados en la conservación de patrimonio histórico, además de expertos en arte urbano.

En cuanto a los espacios, esta Comisión debía tener en cuenta ciertos factores relativos al impacto visual del mural y otros vinculados a la conservación, como la seguridad estructural o estanqueidad del muro (Concejalía de Patrimonio Histórico 2014).
Cada una de las superficies propuestas fueron estudiadas por técnicos municipales, que emitieron un informe sobre seguridad, conservación, impermeabilidad y otras cuestiones técnicas.

Con objeto de mejorar la conservación de los murales, el Ayuntamiento de Vigo solicitó un nuevo estudio en 2017. Fueron analizados entonces diversos aspectos de interés y se tomaron medidas tales como visitas a las viviendas vinculadas para localizar posibles humedades y actuar en consecuencia. También se limpiaron las fachadas y se les aplicó fungicida.

El informe señala la necesidad de aplicar una imprimación sobre los elementos metálicos presentes en algunas fachadas. Además, todas las superficies de las medianeras debían ser pintadas con un tipo de pintura determinado. Una vez pintados los murales fueron protegidos con una capa de barniz. El objetivo era aportar una protección para "prevenir un menor ensuciamiento, un mejor envejecimiento y por tanto una mayor duración de los mismos" (Sánchez de Haz 2017:16). En este proyecto se incluye la aplicación de barniz protector a los murales realizados en las fases anteriores.

En cuanto a los materiales empleados para pintar, son muy dispares. En la mayoría de ocasiones se han usado pinturas no diseñadas específicamente para la creación artística; en su lugar, los artistas han utilizado diversos materiales diseñados inicialmente con fin decorativo o industrial. Por este motivo es imprescindible que el plan de conservación preventiva incorpore una ficha que identifique y describa todos los materiales presentes en cada uno de los murales.

Por señalar tan sólo algunos ejemplos de entre los más de 60 artistas participantes hasta este momento, indicaremos que Alejandro Fernández, creador del mural de O Calvario, explica que para su obra empleó tanto spray como pintura al agua para exteriores. Gonzalo Alonso por su parte usó acrílicos para su mural de la calle Zaragoza 55. El artista Popay usó colores en spray para el mural de la Avenida Atlántida; Elara Elvira y Ana Santiso utilizaron, en su última creación, una pintura plástica para interior y exterior con fungicida en su composición y Magalhaes se atrevió con la materia de carga en su mural de Abeleira Menéndez.

\section{Conservación}

Dado que el proceso de deterioro continúa avanzando se sugiere la elaboración de un plan de conservación preventiva desde la óptica del profesional de conservación restauración de bienes culturales.

El Grupo de Trabajo del Ge-IIC propone la consideración del arte urbano como Bien Cultural Común, BIComún, dada la dificultad de encontrar un encaje legal apropiado que facilite la adopción de medidas para su conservación. Efectivamente, la figura jurídica que otorga el máximo nivel de protección es el Bien de Interés Cultural, BIC, y 
su definición no abarca este tipo de manifestaciones. El BiComún por su parte, es una propuesta reciente que tiene como fin designar a un patrimonio que merece ser conservado como consecuencia de una reflexión colectiva de la sociedad (Masaguer y Vázquez 2014:156).

En el programa redactado por la Concejalía de Patrimonio Histórico de Vigo (2014) se señala como objetivo "conseguir que la ciudadanía se involucre en la conservación y estética de las zonas de goce de todos y del patrimonio", por lo que parece oportuno extender al muralismo esta cobertura. Por tanto, de forma previa al desarrollo del diseño del plan de conservación sería necesario constatar la existencia de esta identificación por parte de los diferentes actores implicados.

Este tipo de obras se multiplican en ciudades de todo el mundo $y$, hayan sido hechas o no con los permisos necesarios, frecuentemente son bien acogidas gracias a una serie de valores que la sociedad percibe en ellas (estético, turístico, social...). En el plano turístico, por ejemplo, los murales de Vigo tienen un mapa-guía específico para su visita y se han creado una web y perfiles para difusión en distintas redes sociales.

En definitiva, es un arte sobre el que se percibe un deseo social de conservación pero que está destinado a ser disfrutado fuera del controlado ambiente del museo, lo que supone un gran reto para los profesionales encargados de su cuidado. A este reto los profesionales responden con nuevos estudios y propuestas dirigidos a solventar la problemática específica que se plantea. Uno de los primeros pasos en este sentido ha sido el desarrollo de un código deontológico de trabajo específico: los profesionales de la conservación restauración han adoptado de forma tácita el propuesto porE.C.C.O. para el desempeño de su trabajo. Por las características particulares que envuelven al arte urbano, el Grupo de Trabajo del Ge-IIC para este campo ha considerado necesario crear un código propio que oriente a los profesionales que trabajen con este tipo de bien (2016:188-192). Esta normativa, como su homóloga de ECCO, es un conjunto de artículos que recoge los principios generales para la aplicación del Código (arts. 1-23) y las obligaciones del autor, propietario o guardián legal (arts. 24-27).

\section{-Perspectivas de conservación}

Buena parte de los artistas participantes en este proyecto comparten el deseo de conservación de las obras, como la pintora Elisa González, quien mostró su disgusto a través de la prensa al saber que su obra quedaría oculta tras la construcción de un edificio en el solar adyacente (Sotelino 2018).

Existen excepciones como Gonzalo Alonso, quien confía en que su mural no vaya a ser afectado por actos vandálicos y cree que, tras una estimación de unos 20 años de vida, "está bien que se deshaga y se pudra porque el arte ha de ser efímero" (Rodríguez 2015). Esta disparidad de pareceres obliga a que el plan de conservación preventiva tenga en cuenta también la opinión de cada creador. Y finalmente, como se ha visto, la Administración tiene la voluntad de que los murales duren en el tiempo, así lo ha expresado el alcalde de la ciudad [3]y como consecuencia se tomaron algunas medidas con este fin (descritas en el apartado 5).

\section{-Agentes de alteración}

A pesar de la amplitud geográfica en la que están distribuidos los murales, éstos están expuestos a una serie de agentes y/o riesgos comunes de sobra estudiados por los profesionales de la conservación restauración, por lo que tan sólo se describirán brevemente.

El plan de conservación preventiva debe conocer tanto estos agentes como aquellos que de forma específica pongan en riesgo cada mural y debe evaluar su efecto periódicamente con el fin de establecer diagnósticos y planes de actuación.

\section{a) Factores intrínsecos}

Dentro de los factores intrínsecos recogeremos aquellos que están directamente vinculados a la creación de las obras.

\section{Soporte}

En este apartado se incluyen los riesgos inherentes a la construcción como morteros, humedades e incluso debilidades estructurales del inmueble. Como soporte debe incluirse no sólo el muro, sino también otros elementos presentes en la fachada (puertas, ventanas, tuberías, rejillas...) con su propio deterioro y amenazas de conservación (manipulación, uso...). Será preciso realizar un mapa de identificación de zonas sujetas a riesgo potencial, sobre las que hacer especial vigilancia (tuberías, posibles desprendimientos...).

En el mural Kraken, realizado por el artista Delio Rodríguez en el año 2017 apreciamos ya la aparición de unas manchas blancas que, con la observación realizada desde la distancia, parecen eflorescencias.

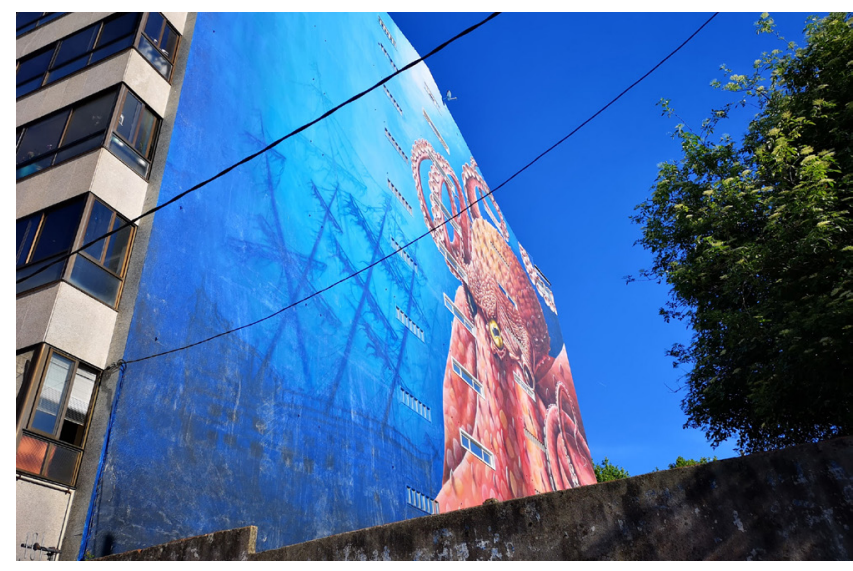

Figura 1.- Manchas blanquecinas en la esquina inferior izquierda del mural Kraken. A. Fernández 


\section{Técnica}

Los materiales y técnicas empleados son de gran importancia para la conservación. El uso de materiales de escasa durabilidad, en mal estado o el uso de técnica inadecuada/incompatible con los materiales del soporte pueden dar como resultado un variado número de alteraciones, al margen de la interacción con el resto de factores que puedan afectar a la conservación del mural. Un ejemplo de ello es el mural Verne, en donde ya hay grandes áreas de pintura levantada, con la incompatibilidad de materiales entre pintura y capa de soporte como causa probable [figura 2].

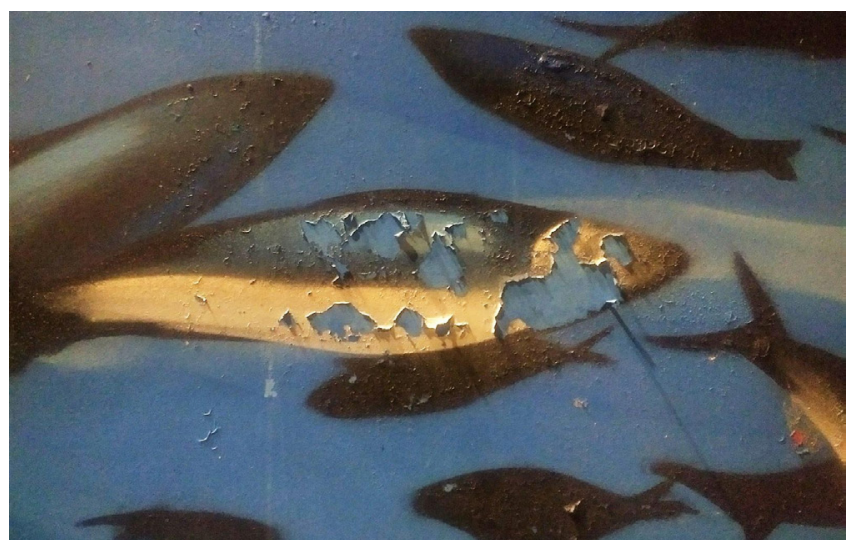

Figura 2.- Levantamiento de película pictórica en el mural de Verne. A. Fernández

\section{b) Factores extrínsecos}

Los factores extrínsecos son aquellos ajenos a la obra que inciden directa o indirectamente en su conservación. Parte de los parámetros que se evaluarán en este apartado son consecuencia del clima de la ciudad, por ello parece necesaria una pequeña descripción de las circunstancias climáticas de Vigo. El clima de esta ciudad es de tipo oceánico, lo que implica una abundante pluviometría en los meses de otoño e invierno y una temperatura en general suave, que oscila entre $5^{\circ} \mathrm{C}$ y $10^{\circ} \mathrm{C}$ en invierno, y $25^{\circ} \mathrm{C}$ y $30^{\circ} \mathrm{C}$, en verano.

\section{Humedad}

Vigo es una zona con una media de precipitaciones al año de 1303 mm. Además del agua de lluvia que impactará directamente sobre la pintura con mayor o menor fuerza, hay que tener en cuenta la humedad ambiental y el agua procedente de la propia estructura del inmueble (tuberías interiores o exteriores en mal estado, sistemas de desagüe en mal estado o insuficientes) que afecten a la obra por capilaridad o incluso por escorrentía.

\section{Temperatura}

La temperatura se verá condicionada por dos factores fundamentales: por una parte, la temperatura ambiental, que en Vigo es por lo general suave; por otra parte, hay que tener en cuenta la temperatura que puede alcanzar la superficie del mural a causa de la incidencia de la radiación solar. Esta incidencia dependerá a su vez de varias circunstancias: la variación según el momento del día y la época del año, y la orientación de la medianera.

\section{Luz.}

La principal exposición de los murales es, lógicamente, de luz solar. Los valores máximos de insolación en el hemisferio norte son en junio y julio y la menor exposición ocurre de diciembre a enero. Pero además hay que tener en cuenta la climatología para determinar el grado de insolación. El clima de Vigo, extensivo a todas las Rías Baixas, implica una atmósfera más limpia y con menos nubes, lo que supone un aumento de la radiación, significativamente mayor que en otras urbes de Galicia (Pettazzi y Salsón 2011:21). También es necesario tener en cuenta la luz artificial de las farolas, especialmente cuando el foco de iluminación está próximo a la superficie del mural y por tanto la incidencia y su efecto es mayor.

\section{Biodeterioro.}

El biodeterioro es la alteración indeseada e irreversible de los materiales causada por organismos o microorganismos. En el caso de los murales de Vigo el principal problema para la conservación tiene variado origen:

1. Animal. En este sentido es posible determinar un daño más acusado por mamíferos en las zonas bajas de los murales y por aves en las zonas altas. En ambos casos el principal problema será la deposición de excrementos/ orines en la superficie. También hay que tener en consideración el biodeterioro por insectos, especialmente a través de la deposición de excrementos.

2. Vegetal. Muchos de los murales están en contacto directo con zonas en las que crecen plantas creando un riesgo de invasión, como ya ocurre en el mural Sea Walls for Oceans [figura 3]. Pero los murales también pueden verse afectados por otros agentes vegetales causantes de biodeterioro, como musgos, líquenes... Tanto su ubicación exterior como las irregularidades de la superficie son factores propicios para el asentamiento vegetal de distinta entidad.

\section{Contaminación.}

Vigo es una ciudad industrial. En el estudio ambiental sobre Pontevedra entre 2004-2017 se concluye que las emisiones contaminantes han dismiuido debido a los efectos de la crisis. Aún así destaca la presencia de contaminantes: flúor, cloruro de hidrógeno, ácido sulfúrico, formaldehídos, sulfuro de hidrógeno, metano y compuestos orgánicos volátiles (Consellería de Medio Ambiente, Territorio e Vivenda, 2017:16). El estudio también apunta a los incendios forestales como causa de la elevada cifra de CO en 2017 (Consellería de Medio Ambiente, Territorio e 


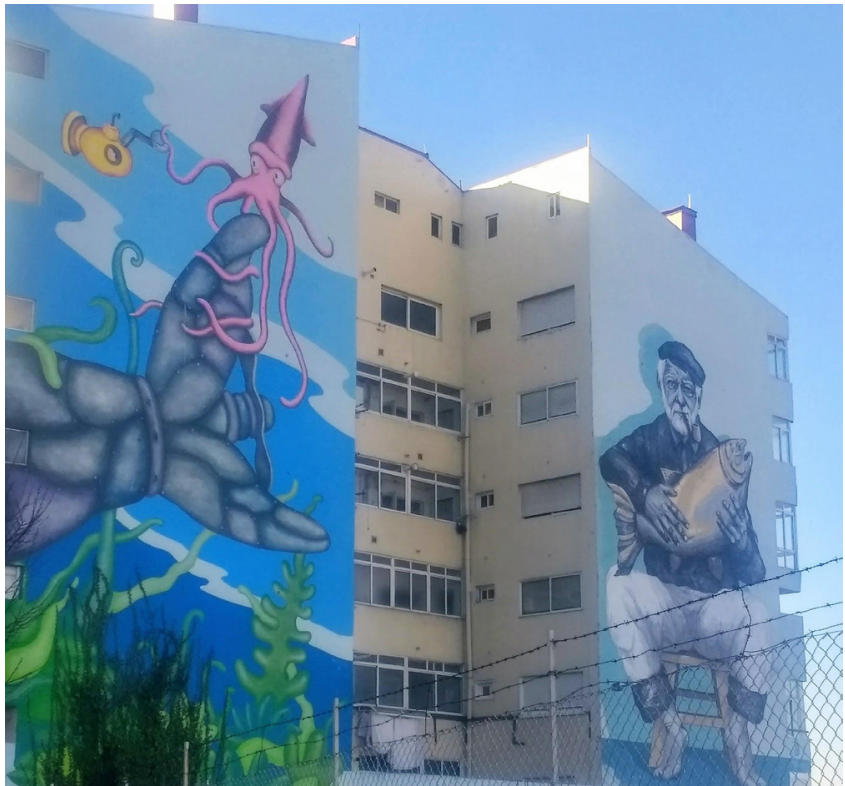

Figura 3.- Mural Sea Walls for Oceans e invasión de la pintura por enredaderas. A. Fernández

Vivenda, 2017:228). Por supuesto también hay que tener en consideración la alta densidad del tráfico como origen de la contaminación ambiental y el impacto que tendrá en murales especialmente expuestos, como el mural del túnel de Beiramar hecho por Lidia Cao. La contaminación unida a la lluvia puede provocar lluvia ácida. El pH de la lluvia se controla de forma habitual y el valor medio para Vigo en el último informe oficial disponible es de 6,6 (Consellería de Medio Ambiente, Territorio e Vivenda, 2017:184).

\section{Viento.}

La importancia del viento radica especialmente en que vaya acompañado de lluvia o partículas sólidas, pudiendo entonces tener un efecto más agresivo sobre las pinturas. Incluso puede facilitar la alteración provocada por aerosoles marinos, especialmente en las fachadas más expuestas. El mural de Lula Goce, todavía íntegro, es uno de los que corre riesgo de verse alterado por la erosión propiciada por el viento, como podemos ver en una fachada próxima y de orientación análoga [figura 4].

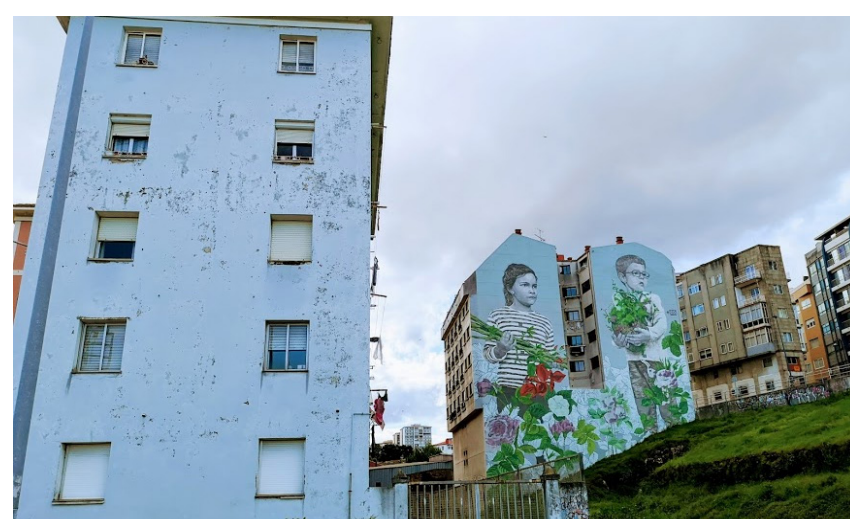

Figura 4.- Mural de Lula Goce en segundo plano y fachada con idéntica orientación y exposición a factores ambientales, en primer plano. A. Fernández

\section{Factor antrópico.}

El daño causado por el ser humano sobre los murales tiene dos posibles orígenes: un daño consciente o el daño inconsciente por acción u omisión. Como actos vandálicos encontramos chicles adheridos, pegatinas o arañazos, y sobre tdo graffitis o grafismos localizados fundamentalmente a metro y medio del suelo, es decir, a una altura cómoda para generar el daño. En la prensa local se hacen eco periódicamente de los graffitis de mayor tamaño (Faro de Vigo 2019) y en general se pasa por alto el gran número de pequeñas pintadas existentes. También hay daño antrópico inconsciente, como los roces casuales. Por ejemplo, en el mural del parque infantil de Navia de Proyecto Ewa, en el que el mural acaba en un banco ampliamente usado y por tanto el roce con la pintura es habitual [figura 5].

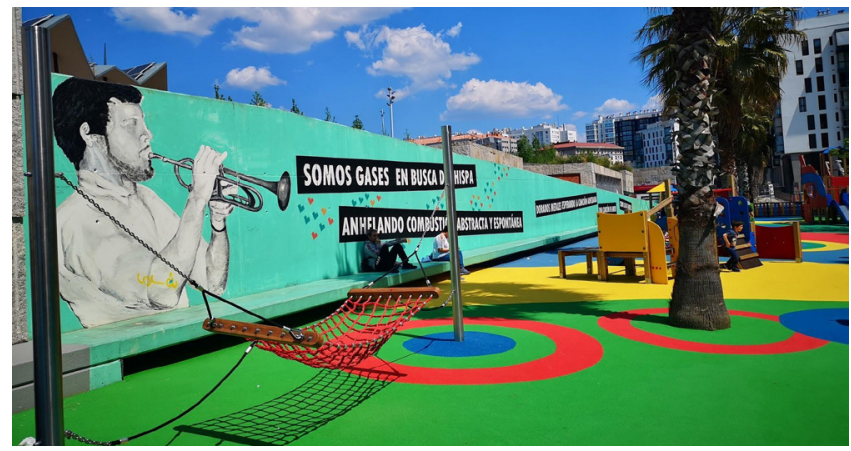

Figura 5.- Ciudadanos apoyados sobre el mural de Proxecto Ewa. A. Fernández

En esta sección podríamos incluir los roces realizados sin querer por automóviles, como en el mural Mulleres da conserva de Beiramar 177 [figura 6].

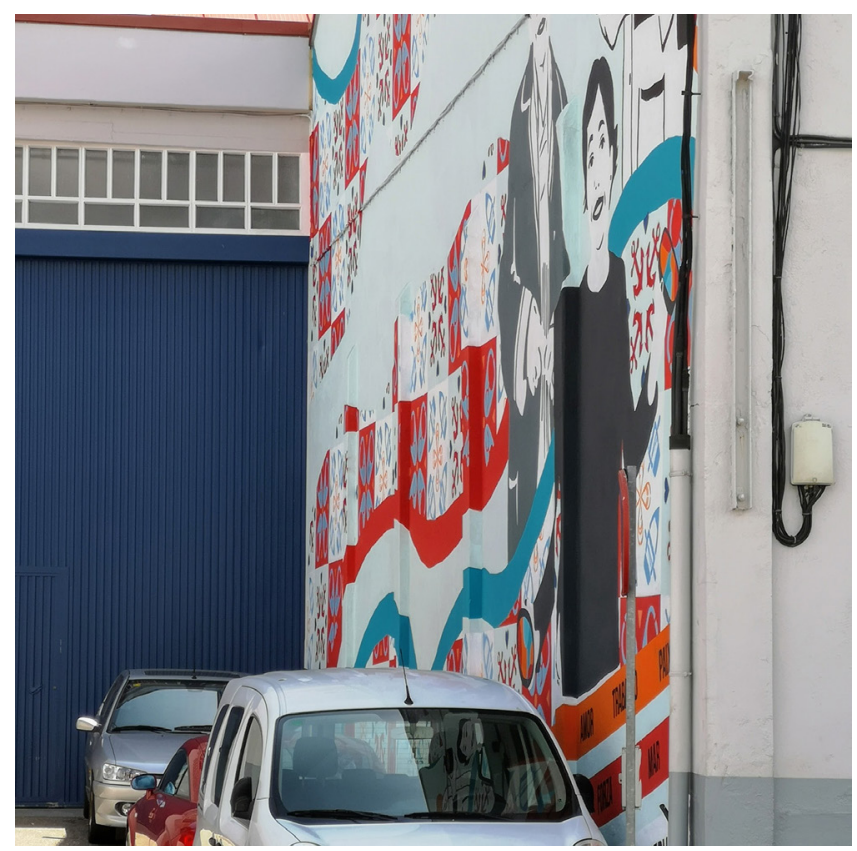

Figura 6.- Coches aparcados junto al mural Mulleres da conserva. A. Fernández 
VIII. Vibraciones.

Vigo no es una ciudad de especial riesgo sísmico. En cambio, sí cabe esperar riesgo derivado de vibraciones transmitidas desde el suelo en aquellas zonas afectadas por una gran densidad de tráfico, especialmente cuando éste es más pesado, de tipo industrial.

\section{Valoración del estado de conservación y diagnóstico}

El número, tamaño y dispersión de las obras obligan a adoptar una metodología específica de trabajo que facilite tanto la toma de datos como su análisis y la determinación de la urgencia a la hora de tomar medidas. Por ello se propone un sistema de recogida de información mediante tablas[3].

Para daños presentes en los murales, se empleará la Tabla 1 que determina la extensión y gravedad del daño sobre el mural. En la Tabla 2 se recoge en cambio la probabilidad de que aparezca un daño todavía no presente y su gravedad una vez aparezca.

Se usará otra tabla para valorar la urgencia con que los daños detectados deban ser intervenidos. El modelo que se propone [Tabla 3] ofrece 3 posibilidades: sin necesidad de intervención, intervención inmediata o necesidad de intervención no inmediata, programable en el tiempo. De este modo se podrá facilitar la organización de las intervenciones y optimizar los recursos. No hay que olvidar que los murales están dispersos en una gran área y que pueden necesitar una importante infraestructura para abordar su intervención, en caso de que sea necesaria.

Finalmente, una tabla con datos básicos de cada mural [Tabla 4] recogerá las medidas de impacto o riesgo de daño de los distintos agentes señalados en el apartado 5.2.

Tabla 1.- Impacto de daño

\begin{tabular}{|c|c|c|c|c|}
\hline DAÑO EXISTENTE & Leve (1) & Medio (2) & Alto (3) & Mry allo (4) \\
\hline Extensión / Impacto & & & & \\
\hline $\begin{array}{c}\text { Extension 0-10\% } \\
\text { Baja (1) }\end{array}$ & $\begin{array}{c}1+1 \\
2\end{array}$ & $\begin{array}{c}1+2 \\
3\end{array}$ & $\begin{array}{c}1+3 \\
4\end{array}$ & $\begin{array}{c}1+4 \\
5\end{array}$ \\
\hline $\begin{array}{l}\text { Extensión } 10-25 \% \\
\quad \text { Media (2) }\end{array}$ & $\begin{array}{c}2+1 \\
3\end{array}$ & $\begin{array}{c}2+2 \\
4\end{array}$ & $\begin{array}{c}2+3 \\
5\end{array}$ & $\begin{array}{c}2+4 \\
6\end{array}$ \\
\hline $\begin{array}{c}\text { Extensión } 25-50 \% \\
\text { Altu (3) }\end{array}$ & $\begin{array}{c}3+1 \\
4\end{array}$ & $\begin{array}{c}3+2 \\
5\end{array}$ & $\begin{array}{c}3+3 \\
6\end{array}$ & $\begin{array}{c}3+4 \\
7\end{array}$ \\
\hline $\begin{array}{c}\text { Extensión } 50-100 \% \\
\text { Mry alta }(4)\end{array}$ & $\begin{array}{c}4+1 \\
5\end{array}$ & $\begin{array}{c}4+2 \\
6\end{array}$ & $\begin{array}{c}4+3 \\
7\end{array}$ & $\begin{array}{c}4+4 \\
8\end{array}$ \\
\hline & Categoria 4 & Categoria 3 & Categoria 2 & Categońa 1 \\
\hline
\end{tabular}

Tabla 2.- Riesgo de aparición de daño

\begin{tabular}{|c|c|c|c|c|}
\hline DAÑO PROBABLE & Leve (1) & Medio (2) & Alto (3) & Muy allo (4) \\
\hline Probabildad / Impacto & & & & \\
\hline $\begin{array}{c}\text { Improbable que se produzca } \\
\text { Hesgo bajo (1) }\end{array}$ & $\begin{array}{c}1+1 \\
2\end{array}$ & $\begin{array}{c}1+2 \\
3\end{array}$ & $\begin{array}{c}1+3 \\
4\end{array}$ & $\begin{array}{c}1+4 \\
5\end{array}$ \\
\hline $\begin{array}{l}\text { Es posible que se produzca } \\
\text { Fiesgo medio (2) }\end{array}$ & $\begin{array}{c}2+1 \\
3\end{array}$ & $\begin{array}{c}2+2 \\
4\end{array}$ & $\begin{array}{c}2+3 \\
5\end{array}$ & $\begin{array}{c}2+4 \\
6\end{array}$ \\
\hline $\begin{array}{l}\text { Muy probable que se produzca } \\
\text { Fiesgo allo (3) }\end{array}$ & $\begin{array}{c}3+1 \\
4\end{array}$ & $\begin{array}{c}3+2 \\
5\end{array}$ & $\begin{array}{c}3+3 \\
6\end{array}$ & $\begin{array}{c}3+4 \\
7\end{array}$ \\
\hline $\begin{array}{l}\text { Seguro que se produce, } \\
\text { incluso con inmediatez } \\
\text { Fesgo muy alto (4) }\end{array}$ & $\begin{array}{c}4+1 \\
5\end{array}$ & $\begin{array}{c}4+2 \\
6\end{array}$ & $\begin{array}{c}4+3 \\
7\end{array}$ & $\begin{array}{c}4+4 \\
8\end{array}$ \\
\hline & Categoria 4 & Categoría 3 & Categoria 2 & Categoriz 1 \\
\hline
\end{tabular}


Tabla 3.- Urgencia de intervención

\begin{tabular}{|c|l|l|l|}
\hline \multicolumn{1}{|c|}{ URGencia } & \multicolumn{1}{|c|}{$\mathbf{0}$} & \multicolumn{1}{c|}{$\mathbf{1}$} \\
\hline $\begin{array}{l}\text { Urgencia de la intervención con la que debe } \\
\text { ser afrontada una alteración }\end{array}$ & $\begin{array}{l}\text { No precisa } \\
\text { intervención }\end{array}$ & $\begin{array}{l}\text { Precisa intervención } \\
\text { pero no es urgente }\end{array}$ & $\begin{array}{l}\text { Precisa } \\
\text { intervención } \\
\text { inmediata }\end{array}$ \\
\hline
\end{tabular}

Tabla 4.- Recolección de datos

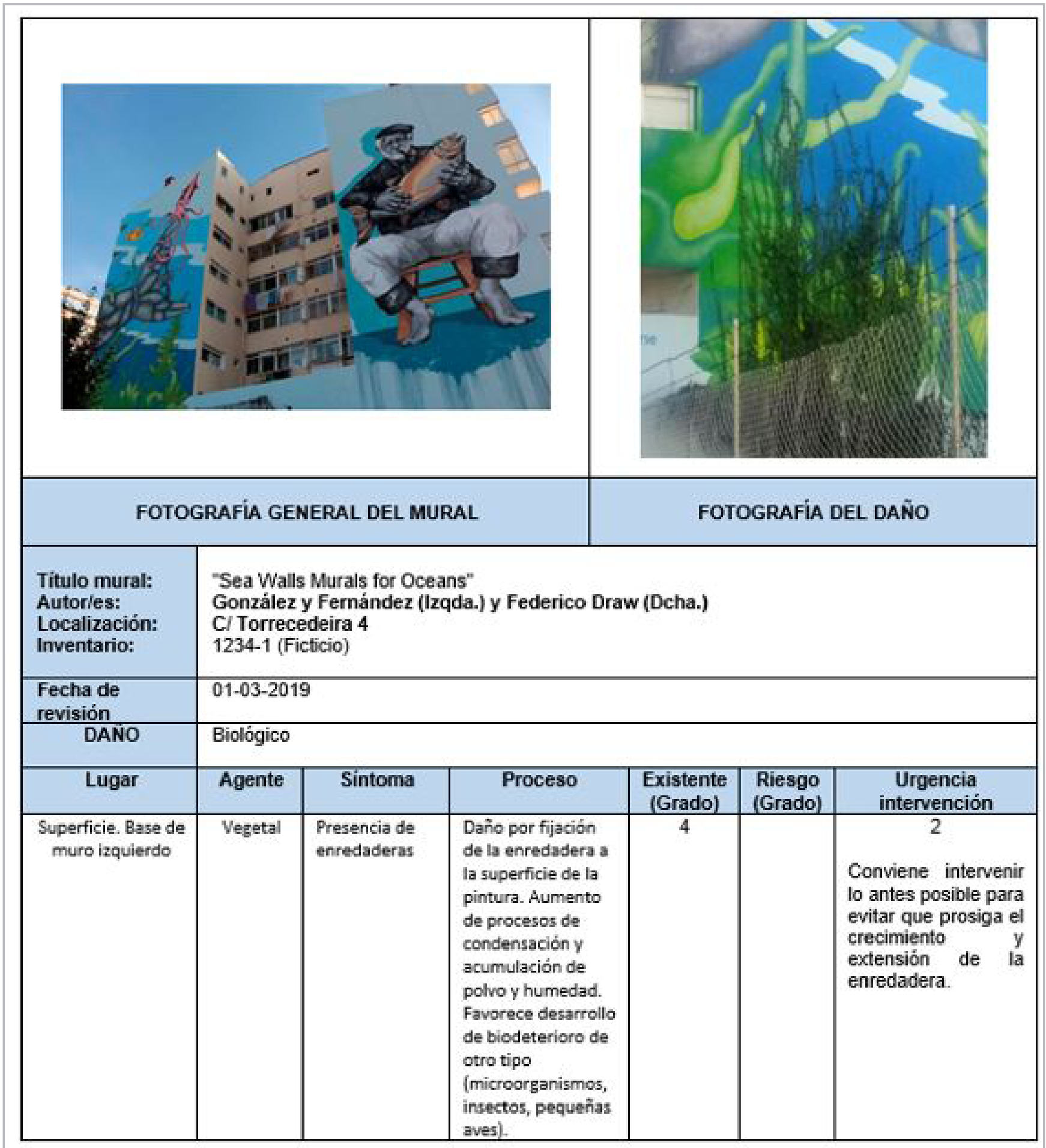


Se recomienda evaluar anualmente la incidencia de todos los agentes señalados. De este modo se podrán establecer diagnósticos singularizados de daños o de riesgo de aparición de los mismos y, en consecuencia, determinar los tratamientos necesarios y el orden de prioridad para su aplicación.

\section{Otras medidas}

La preocupación por la conservación preventiva no es nueva. En el año 2000 el ICCROM animó a centrar las acciones sobre el patrimonio cultural en la prevención de los riesgos como una forma más eficaz y sostenible de gestión del patrimonio, y así lo recoge la Resolución de la Reunión de Vantaa. Este proyecto se realiza inspirado en esas directrices.

Este plan consta de dos apartados: uno dedicado a las medidas previas a la ejecución de los murales y otro para los ya pintados.

\section{a. Medidas para obras que van a ser realizadas}

Esta fase cuenta con la ventaja de poder influir en la toma de decisiones que puedan favorecer la conservación del mural a ejecutar. Las medidas serían las siguientes:

1. Inclusión de un profesional de conservación restauración en el Comité de selección de superficies, con el fin de señalar las más problemáticas en términos de conservación.

2. Propuesta y ejecución de medidas de conservación preventiva para cada una de las superficies elegidas.

3. Oferta de asesoramiento de técnicas y materiales, para el/los artistas que lo deseen.

\section{b. Plan de conservación preventiva para murales ejecutados.}

1. Documentación. La recogida de información relativa a los murales es de gran importancia, especialemente teniendo en cuenta que en algunos casos el deterioro y la pérdida del mural serán inevitables y la documentación será todo el legado que quede tras su desaparición. En este sentido se proponen las siguientes medidas:
a. Asignación de un número de inventario.
b. Elaboración de ficha documental de cada obra. Para este fin se propone el uso del Modelo de registro elaborado por el Grupo de Trabajo de Arte Urbano del Ge-IIC (Úbeda 2016:175-176).
c. Creación de una base de datos que recoja toda la información de cada mural, para facilitar su gestión.

2. Revisión anual de cada uno de los murales con las tablas propuestas en el apartado 7.

3. Posteriormente, se tomarán medidas apropiadas para las incidencias detectadas. Éstas serán realizadas por profesionales de la conservación restauración en el ámbito de su competencia o bien serán sugeridas a la administración para que pueda proceder a su encargo. Por ejemplo:

a. Medidas a realizar profesionales de la conservación restauración tras la detección del problema:

i. Eliminación vegetación invasora sobre los murales.

ii. Tratamiento de daños causados por humedad.

b. Medidas a desarrollar por otros profesionales, con la supervisión del responsable del plan:

i. Prevención de asentamiento de vegetación invasora en solar adyacente.

ii. Mejora del sistema de evacuación del agua de lluvia en el túnel de Beiramar, para evitar que forme charcos y ascienda por capilaridad en el mural, como sucede en la actualidad.

4. Se propone contar con la colaboración activa de la sociedad en la vigilancia de los murales. Se considera oportuno contar con un colaborador en el propio inmueble (podría tratarse de un representante de la comunidad de vecinos o de la asociación vecinal) que dé aviso en caso de incidencias.

5. Plan de calidad. Como todo proyecto que se pretenda mantener en el tiempo, el propio plan necesitará una evaluación periódica de calidad que permita detectar fallos, aplicar de medidas correctivas e implantar mejoras.

Es preciso señalar que muchas soluciones de conservación que se propongan deberán pasar por un comité multidisciplinar del Ayuntamiento que valore la compatibilidad de las propuestas con otras necesidades/ usos del entorno.

\section{Conclusiones}

Vigo, como otras muchas ciudades a nivel mundial, se ha dejado seducir por la estética y el diálogo con la sociedad que establecen los murales. El gran número de medianeras que afeaban la ciudad ha resultado ser la excusa perfecta y el lienzo ideal para este proyecto, que ya consta de más de 70 obras.

En este caso existe un especial interés de la administración y la sociedad por su preservación; sin embargo, los murales de Vigo. Ciudad de color no están protegidos por las leyes de patrimonio, nacionales o autonómicas. Estaríamos ante uno de esos elementos que podrían ser englobados bajo la denominación de BIComún.

Este plan pretende proteger los murales creados hasta ahora y todos aquellos que se incorporen en futuras fases. Está basado en la asunción de un código ético específico, así como en el uso de una metodología concreta de trabajo que facilita la recogida de datos relativos al estado de conservación, facilitando la evaluación de riesgos y 
prioridades y permitiendo una adopción razonada de decisiones.

Los factores decisivos para la conservación de los murales mantienen algunas similitudes con los que afectan a otro tipo de bienes en entornos mejor conocidos. Sin embargo, en nuestro caso, algunos de los parámetros no son en absoluto manipulables (temperatura, humedad, por ejemplo), lo que obliga a un trabajo alternativo que permita, a pesar de las dificultades, retrasar o disminuir sus consecuencias.

Otra ventaja de este sistema es que optimiza el uso de las infraestructuras necesarias, tanto para revisión como para intervención y lógicamente ello redundará en un mejor empleo de los recursos disponibles.

Este sistema de recolección de información, sostenido en el tiempo, facilitará también conocer la evolución de muchos materiales empleados en la actualidad para la creación, pero de los que todavía carecemos de información suficiente, permitiendo mejorar el pronóstico de conservación de estas obras en el futuro.

Es preciso destacarquees desdela conservación restauración de bienes culturales que se pueden dar las soluciones apropiadas a los retos que supone la conservación de estos murales. Por ello, conviene avanzar en las líneas de investigación recientemente abiertas en este campo.

\section{Notas}

[1] Url del proyecto Vigo. Cidade de cor: http://ciudaddecolor.vigo. org/

[2] En 2015 se invirtieron $230.000 €, 224.000 €$ en 2016, 297.043,71 $€$ en 2017 y $298.582,52 €$ en 2018 , según se desprende de en las licitaciones de los expedientes, 8185-307, 8521-307, 8845-307 que se pueden consultar a través de la web del ayuntamiento www.vigo.org

[3] En declaraciones recogidas por el mismo artículo de Sotelino, el Alcalde de Vigo indicaba que los murales recibirían "un tratamiento especial para garantizar su durabilidad y preservarlos así en buen estado de conservación durante al menos 100 años".

[4] Para el diseño de las tablas se han tomado como referencia las tablas propuestas por Carrera (2018) para la elaboración de planes de conservación preventiva en yacimientos arqueológicos, por la similitud de diversos parámetros como exposición a factores ambientales no controlables, dispersión geográfica, etc. Éstas tienen a su vez su base en el sistema de evaluación de riesgos diseñado por Culubret et al. (2008:28-29), que lógicamente también se ha tenido en consideración. Así, las asignaciones numéricas funcionan del mismo modo que aquellas: se asignan valores de 1 a 4 según si el riesgo de aparición o la gravedad del daño es leve (1), media (2), alta (3) o muy alta (4), y los posibles resultados de las sumas oscilan entre 2 y 8 . Se genera así la posibilidad de agrupar daños en 4 categorías, siendo la categoría 1 la que indica la mayor gravedad y 4 , la que menos.

\section{Bibliografía}

CARRERA RAMÍREZ, F. (2018): Conservación preventiva de yacimientos arqueológicos: ¿empezamos? VI Congreso GEIIC. 20-22 Septiembre 2018. Vitoria, 376-384.

CHATZIKADIS, M. (2018): Preventive conservation and monitoring of Street art, graffiti, and public murals: education and training as an effective tool. En Actas VI Congreso Ge-IIC. pp. 99-106 Madrid, 2018. Disponible en <https://www.ge-iic.com/2018/10/03/actasdel-vi-congreso-del-geiic/ > [Consulta: 8 de febrero 2019]

CIANCABILLA, LUCA (2015): The sight gallery: salvaguardia e conservazione della pittura murale urbana contemporanea a Bologna. Bolonia, Bolonia University Press.

CONCELLERÍA DE PATRIMONIO HISTÓRICO (2014): Programa para arrumbar medianeiras e outros elementos verticais con incidencia sobre a vía pública, e demais espazos libres que conforman a paisaxe urbana. Vigo, Concello de Vigo. Disponible en vigo.org

CONSELLERÍA DE MEDIO AMBIENTE, TERRITORIO E VIVENDA (2017): Informe anual da calidade do aire de Galicia 2017. Santiago de Compostela, Xunta de Galicia.

CULUBRET WORMS, B., Hernández Azcutia, M., Hidalgo Cámara, E., Martínez de Marañón Yanguas, M. y Rallo Gruss, C. (2008): Guía para un plan de protección de colecciones ante emergencias. Madrid, Ministerio de Cultura.

GARCÍA GAYO, E. (2015): "Introducción". Mural Street Art Conservation. Observatorio del Arte Urbano no 1 pp. 4-5. <https:// issuu.com/observatoriodearteurbano/docs/mural 1> [Consulta: 8 de enero 2019]

GRUPO DE ARTE URBANO (2016): “Anexo I. Propuesta de código deontológico para la conservación y restauración de arte urbano" en Ge-Conservación no 10. Monográfico Arte Urbano. Conservación y Restauración de Intervenciones Contemporáneas. pp. 188-192 $<$ http://observatoriodearteurbano.org/wordpress/wp-content/ uploads $/ 2017 / 01 / \mathrm{Ge}$-conservacio\%CC\%81n-N\%C2\%BA10Suplemeto-Arte-Urbano.pdf> [Consulta: 3 de abril de 2019]

LUQUE RODRIGO, L. (2015). "Arte urbano para la ciudadanía: conservación colectiva".MuralStreetArtConservation.Observatoriodel ArteUrbano n ${ }^{\circ} 1$ p. $13<$ https://issuu.com/observatoriodearteurbano/ docs/mural 1> [Consulta: 8 de enero 2019]

MASAGUER OTERO, M. y VÁZQUEZ VEIGA, A. (2014): "BIComun: un experimento en el espacio público" Tejuelo: Didáctica de la Lengua y la Literatura. Educación, no 19, págs. 154-158. < $\underline{\text { https:// }}$ dialnet.unirioja.es/servlet/articulo?codigo $=4725343>$ [Consulta: 1 de marzo 2019]

MATA DELGADO A. L. (2013) “Conservando el street art \& grafiti, la pertinencia de su conservación y la problemática material derivada de su técnica de manufactura". Memorias del X Foro Académico ECRO, México, 2013. Documento electrónico disponible en http:// www.ecro.edu. $\mathrm{mx} /$ memorias $\mathrm{x}$ foro.html 
MÉNDEZ, ÓSCAR (2017): “Vigo llegará a las 100 medianeras en un lustro y aspira a ser la urbe con más murales de Europa" en Faro de Vigo, 12 de julio. <https://www.farodevigo.es/granvigo/2017/07/12/vigo-llegara-100-medianeras-lustro/1714993. html > [Consulta: 14 de abril 2019]

PASTORIZA, JAVIER (2014): "La calle Príncipe estrenará el primer mural en un edificio con la réplica de "Fiestra" de Lugrís" en Faro de Vigo, 22 de agosto. <https://www.farodevigo. es/gran-vigo/2014/08/22/calle-principe-estrenara-primermural/1079661.html> [Consulta: 30 de noviembre 2018]

PETTAZZI, A. Y SALSÓN CASADO, S. (2011): Atlas de radiación solar de Galicia. Santiago de Compostela, Ed. Xunta de Galicia. $<$ https://www.meteogalicia.gal/datosred/infoweb/meteo/ docs/publicacions/libros/Atlas Radiacion Solar Galicia.pdf> [Consulta: 16 de abril 2019]

PONCE, CARLOS (2018): "El arte llega a los colegios" en Faro de Vigo, 30 de diciembre. <https://www.farodevigo.es/granvigo/2018/12/30/arte-llega-colegios/2025295.html> [Consulta: 31 de diciembre 2018]

PONCE, CARLOS (2019): “Un mural en una medianera relanzará el "olvidado" barrio vigués de Ribadavia" en Faro de Vigo, 6 de enero. <https://www.farodevigo.es/gran-vigo/2019/01/06/ mural-medianera-relanzara-olvidado-barrio/2028210.html > [Consulta: 7 de enero 2019]

PREGO, CARLOS (2018): "El museo urbano fija a Vigo en el mapa del 'street art'" en Faro de Vigo, 30 de diciembre. $<$ https://www.farodevigo.es/gran-vigo/2018/12/30/museourbano-fija-vigo-mapa/2025294.html> [Consulta: 30 de diciembre 2018]

REDACCIÓN ATLÁNTICO DIARIO (2014): “Príncipe presume ya de su gigantesco mural de Lugrís" en Atlántico Diario, 2 de septiembre. https://www.atlantico.net/articulo/vigo/principepresume-gigantesco-mural-lugris/20140907002612434005. html> [Consulta: 30 de noviembre 2018]

REDACCIÓN FARO DE VIGO (2019): "Las pintadas vuelven a cebarse con uno de los murales de Vigo" en Faro de Vigo, 3 de enero. <https://www.farodevigo.es/gran-vigo/2019/01/03/ pintadas-vandalicas-ceban-gran-mural/2026422.html> [Consulta: 3 de enero 2019]

RODRÍGUEZ, A. (2015): “Un colorista paisaje urbano alegra la calle" en Faro de Vigo, 11 de noviembre. <https://www. farodevigo.es/especiales/arte-urbano/2015/11/coloristapaisaje-urbano-alegra-calle-n861 2 24639.html> [Consulta: 10 de enero 2019]

SÁNCHEZ DE HAZ, B. (2017) "Las actuaciones preparatorias orientadas al desarrollo del $3^{a}$ programa para el pintado de medianeras y otros elementos verticales con incidencia sobre la vía pública" Disponible en http://www.vigo.org/csv empleando el código de verificación 2DE34-D2B88-4EAC4-842BE. [Consulta: 7 de febrero 2018]
SÁNCHEZ PONS, M., SHANK, W. Y FUSTER LÓPEZ, L. (2015): Conservation Issues in Modern and Contemporary Murals. Cambridge Scholars Publishing

SOTELINO, BEGOÑA (2018): "Por mi enfermedad respiratoria, el mural es símbolo del esfuerzo" en La Voz de Galicia, 24 de marzo. < https://www.lavozdegalicia.es/noticia/vigo/vigo/2018/03/24/ exposiciones-formacion-enfermedad-respiratoria-mural-simboloesfuerzo/0003 201803V24C12991.htm> [Consulta: 7 de enero 2018]

ÚBEDA GARCÍA, M. (2016). Propuesta de un modelo de registro para el análisis y documentación de obras de arte urbano. GeConservacióN, , 169-179. Consultado de https:/ge-iic.com/ojs/ index.php/revista/article/view/410 [Consulta: 3 de abril de 2019]

\section{Autor/es}

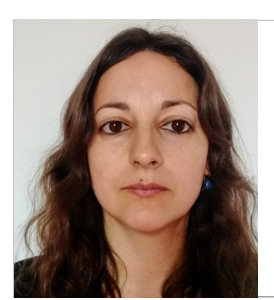

\section{Andrea Fernández Arcos}

andreafarcos@gmail.com ESCRBBCCG

Andrea Fernández Arcos: Titulada en la especialidad de pintura por la Escuela Superior de Conservación y Restauración de Bienes Culturales de Galicia (2003), Máster en Conservación, Restauración y Exposición de Bienes Culturales por la Universidad Complutense de Madrid (2009) y Postgrado en Gestión, Preservación y Difusión de Archivos Fotográficos por la Universitat Autónoma de Barcelona (2014).

Desarrolló su trabajo en instituciones como el Museo Nacional Thyssen-Bornemisza (2004-5), Museo Nacional del Prado (2005-8) o el Museo del Traje-CIPE entre otros (2010-13). Desde 2013 pertenece al Departamento de Conservación y Restauración de Bienes Pictóricos de la Escuela Superior de Conservación y Restauración de Bienes Culturales de Galicia, donde actualmente desempeña el cargo de vicedirección.
Artículo enviado el 06/09/2019 Artículo aceptado el 11/10/2019 